\title{
INFLUÊNCIA DAS VARIAÇÖES CIRCADIANAS E DE TEMPERATURA NO AVEI
}

\author{
Gibran Franzoni Rufca*1, Eduardo Zaffani', Rachel Zerbini', Felipe Franco Pinheiro Gaia², Fabio de Nazaré Oliveira ${ }^{3}$, Waldir Antonio Tognolla ${ }^{4}$
}

Trabalho realizado na Faculdade de Medicina de São José do Rio Preto - FAMERP e no Hospital de Base, São José do Rio Preto, São Paulo

\author{
*Correspondência: \\ Avenida Jamil Feres Kfouri, \\ 250 - Ap 33 - Jardim \\ Panorama \\ CEP 15091-240 -São José \\ do Rio Preto - SP \\ Tel/Fax: (17) 32164084 \\ gvfr@uol.com.br
}

\begin{abstract}
RESUMO
OBjetivos. Acidente vascular encéálico isquêmico (AVEi) representa a terceira maior causa de morte e gastos anuais. A literatura demonstra maior ocorrência no período da manhã e em épocas mais frias, podendo ter uma maior prevenção nestes momentos. Portanto, os objetivos foram identificar os fatores de risco, avaliar a influência das variações circadianas e de temperatura na prevalência do AVEi.

Métodos. Foram estudados 100 pacientes com o diagnóstico de AVEi. O horário de instalação foi analisado em quatro períodos, sendo obtido do paciente ou dos familiares. A temperatura climática foi obtida do INMET.

Resultados. O AVEi foi classificado de acordo com os subtipos estabelecidos pelo projeto TOAST. Dos pacientes, $66 \%$ tinham hipertensão arterial como principal fator de risco. Houve um predomínio de casos no período das 6 h00 às I Ih59 ( $p<0.000 \mathrm{I})$ e após variação negativa da temperatura $(p<0,0001)$.

Conclusãa. Encontrou-se uma forte relação entre a prevalência de eventos encefálicos isquêmicos e variação negativa de temperatura e que o horário de maior frequência foi entre 6 h00 e I I h59. Não foi possível avaliar as variações de temperatura e circadianas para cada subtipo de AVEi, pois o número de casos estudados não permitiu avaliaçãa estatística significante.
\end{abstract}

Unitermos: Acidente cerebral vascular. Ritmo circadiano. Temperatura ambiente. Fatores de risco.

\section{INTRODUÇÃo}

Acidente vascular encefálico (AVE) é a síndrome de origem vascular caracterizada pelo desenvolvimento rápido de sinais e sintomas de uma alteração focal ou global da função cerebral durando mais do que 24h ou levando à morte!. Essa doença representa nos Estados Unidos (EUA), a terceira maior causa de morte com um número anual de 150000 óbitos, sendo que três milhões de pacientes sobrevivem ao AVE. Nos EUA, os custos relacionados ao AVE são estimados em US\$ I 40.000 por paciente, com um custo total de US\$ 62.7 bilhões no ano de $2007^{1,2,3}$. No Brasil, a taxa de incidência é de I 56 casos por 100.000 habitantes, com uma prevalência de 6,2\% e uma taxa de mortalidade de I I casos por 100.000 habitantes $1,2,3$.

Quanto ao prognóstico e reabilitação de pacientes com AVE, para os eventos isquêmicos a mortalidade nos primeiros três meses de diagnóstico é de 15\%. Dos pacientes que sobrevivem ao evento, 33\% a $50 \%$ ficam com sequelas graves e a reabilitação pode durar de meses a anos. Com relação aos eventos hemorrágicos, $30 \%$ a $50 \%$ dos pacientes morrem nos primeiros 30 dias do diagnóstico. $\mathrm{O}$ risco de recorrência do evento é cerca de $25 \%$ 1,2,3.

Os fatores de risco reconhecidos para AVE são hipertensão, estenose de carótida, obesidade, dislipidemia, sedentarismo, etilismo, uso de anticoncepcionais orais, arritmias e cardiopatias, ${ }^{1,23}$. Nesse contexto, de acordo com sua etiologia, o AVE pode ser classificado em isquêmico (AVEi) e hemorrágico (AVEh), sendo o AVEi subdividido, de acordo com sua etiologia, em aterotrombótico, cardioembólico, lacunar, de outras causas e indeterminado ${ }^{1-4}$.

Inúmeros estudos internacionais demonstram associação entre prevalência de AVE e certos períodos do dia e ano. A maioria, indica sua ocorrência no período de $6 \mathrm{~h} 00$ às $12 \mathrm{~h} 00$ e em épocas mais frias do ano ${ }^{5-23}$. A fisiopatologia dessa associação baseia-se nas variações circadianas e de temperatura dos fatores envolvidos no AVEi e no AVEh, como aumento do fibrinogênio, da pressão arterial e agregabilidade plaquetária-23. Logo, mesmo se uma pequena proporçãa dos casos for atribuída à temperatura e ao período, pode-se prevenir os seus efeitos pelo do uso de medidas protetoras nessas condições mais vulneráveis ${ }^{23}$, evitando significativo número de acidentes cerebrovasculares, e podese consolidar o atendimento e o tratamento através de um melhor preparo dos serviços para estes períodos de maior risco ${ }^{21-23}$. Sendo assim, este estudo tem como objetivos identificar os fatores de risco dos pacientes com diagnóstico de AVEi, avaliar a influência das variações circadianas e de temperatura na prevalência do AVEi.

\section{Métodos}

Trata-se de um estudo transversal realizado em 100 pacientes internados no Hospital de Base da Faculdade de Medicina de São José 
do Rio Preto, no período de 14 de maio de 2005 a 15 de março de 2006, com o diagnóstico de AVEi. Dados clínicos e pessoais foram coletados de prontuários e também durante entrevista com os pacientes que concordaram em participar deste estudo mediante assinatura de Termo de Consentimento Livre e Esclarecido conforme o processo 3244/05, de parecer 142/2005, do Comitê de Ética Médica da referida instituição. Como foram selecionados apenas casos com os dados completos, não ocorreram perdas durante o trabalho. Todos os pacientes foram submetidos a exame de tomografia de crânio, eletrocardiograma e radiografia de tórax nas primeiras 24h para diferenciação entre os tipos de AVEi e diagnóstico diferencial com outras doenças, de acordo com o protocolo da entidade. Para confirmação do diagnóstico, 16 pacientes foram submetidos também à ressonância magnética. Em casos selecionados foram realizados também ecocardiograma e duplex de carótidas.

Neste estudo, considerou-se hipertensão arterial (HA) como pressão arterial sistólica maior ou igual a $140 \mathrm{mmHg}$ e diastólica maior ou igual a $90 \mathrm{mmHg}{ }^{24}$; Diabetes melitus (DM) como glicemia de jejum maior ou igual a $126 \mathrm{mg} / \mathrm{dl}$, glicemia de duas horas após $75 \mathrm{~g}$ de glicose maior ou igual a $200 \mathrm{mg} / \mathrm{dl}$ ou glicemia aleatória maior ou igual a $200 \mathrm{mg} /$ dl na presença de poliúria, polidipsia e perda de peso não explicada25; cardiopatia como história de síncope, angina, insuficiência cardíaca diagnosticada anteriormente, fenômenos tromboembólicos sistêmicos, diagnóstico na infância, ritmo de galope (B3), cardiomegalia importante, eletrocardiograma (ECG) com sinais de hipertrofia ventricular esquerda, alterações de repolarização ventricular esquerda, sinais de aumento de átrio esquerdo ou baixa voltagem do complexo QRS26; dislipidemia como colesterol maior que $200 \mathrm{mg} / \mathrm{dl}$ e/ou LDL maior que $100 \mathrm{mg} / \mathrm{dl} \mathrm{e/}$ ou triglicérides maior que $150 \mathrm{mg} / \mathrm{dl}^{27}$; acidente isquêmico transitório (AIT) como um episódio, de origem vascular oclusiva, agudo e focal de perda de função cerebral por um tempo máximo de $24 \mathrm{~h}$ e regredindo após este período ${ }^{28}$; etilismo através dos critérios Diagnostic and Statistical Manual of Mental Disorders, Fourth Edition (DSM-IV), requerendo que três dos oito critérios estejam presentes; tabagismo como uso de mais de 100 cigarros em toda a vida e/ou continua fumando atualmente ${ }^{29}$; fibrilação atrial (FA) como encontro, ao eletrocardiograma (ECG), de uma troca de ondas $P$ consistentes por oscilações rápidas que variam em forma, tamanho e tempo, associadas a uma resposta atrioventricular irregular e rápida ${ }^{30}$.

O horário de instalação foi analisado em quatro períodos de seis horas (00h00h às 05h59h; 6 h00 às I Ih59; I 2 h00 às I7h59 e I 8 h00 às 23h59). O horário de início dos sintomas foi obtido do próprio paciente ou de seus familiares quando o mesmo não pôde se comunicar. Para os pacientes que despertaram com o déficit foi atribuído arbitrariamente 0 horário em que o mesmo foi visto sem sintomas pela última vez (exemplo: ao se deitar).

Os dados referentes à temperatura climática foram obtidos da estação metereológica do INMET (Instituto Nacional de Meteorologia) das seguintes cidades do Estado de São Paulo: Catanduva, Votuporanga e Presidente Prudente. Quando a procedência dos pacientes não era de tais cidades, foram escolhidas as medidas da estação mais próxima da cidade do paciente. Foi utilizada como referência para a variação de temperatura a diferença entre a temperatura do período do dia do evento e a temperatura do mesmo período do dia anterior.
O AVEi foi classificado de acordo com o protocolo usado no projeto TOAST - Trial of Org 10172 in Acute Stroke Treatment.

O teste estatístico Qui Quadrado foi aplicado utilizando-se o GraphPad Prism versão 4.00 para Windows XP, GraphPad Software, San Diego California USA, www.graphpad.com.

\section{Resultados}

Foram estudados consecutivamente 100 pacientes internados no departamento de neurologia do Hospital de Base de São José do Rio Preto. Destes, $50 \%$ eram do sexo feminino e $50 \%$ eram do sexo masculino. A idade média dos pacientes foi de 65,7 anos (DP $= \pm$ 15,93). Os indivíduos estudados tiveram origem das regiões das seguintes cidades: Aparecida d' Oeste, Araraquara, Birigui, Fernandópolis, Jales, José Bonifácio, Limeira, Nova Granada, Ribeirão Preto, São José do Rio Preto e São Paulo, todas do Estado de São Paulo, Frutal e Fronteira, ambas do Estado de Minas Gerais. A análise dos fatores de risco dos pacientes acometidos por AVEi; da distribuição dos tipos de AVEi segundo os critérios de TOAST; da forma de instalação dos AVEis; da variação circadiana da instalaçãa do AVEi; e da variação temperatura do AVEi, nos permitiu chegar aos resultados descritos abaixo.

Os principais fatores de risco identificados pelo estudo se distribuíram da seguinte maneira: HA representou 66\% dos casos; tabagismo 47\%; presença de cardiopatia 20\%; DM 18\%; presença de prótese valvar 9\%; dislipidemia 7\%; FA 7\%; episódio prévio de AIT 3\% e etilismo $1 \%$. Treze por cento dos pacientes não apresentavam fatores de risco e em $2 \%$ dos casos não foi possível obter esta informação.

Os tipos de AVEi segundo os critérios de TOAST são, em nosso trabalho distribuídos da seguinte maneira: $48 \%$ tiveram etiologia aterotrombótica; $30 \%$ cardioembólica; 16\% indeterminada e 6\% lacunar.

Correlacionando os tipos de AVEi e a forma de instalação destes, encontramos que $22 \%$ dos casos de instalação progressiva tiveram origem aterotrombótica e dos casos de instalação súbita, 19\% tiveram origem cardioembólica (Gráico I).

Os casos de AVEi se distribuíram, de acordo com o horário de instalação, com predomínio de casos no período das 6 h00 às I Ih59 $(p<0.000$ I) (Gráfico 2).

Com relação à variação de temperatura, analisando-se a variação de 24h entre o horário do dia do evento e o horário do dia anterior, obtivemos, quanto aos casos de AVEi, que 55\% dos eventos ocorreram após uma variação negativa da temperatura $\left(-1^{\circ} \mathrm{C}\right.$ a $-8,6^{\circ} \mathrm{C} ; \mathrm{p}<$ 0,000 I) (Gráfico 3).

\section{Discussão}

Neste estudo, destacam-se entre os fatores de risco para AVEi: hipertensão arterial, tabagismo, diabetes e cardiopatias. Sendo a hipertensão arterial o principal fator de risco (66\%), comparável ao estudo de Oliveira et al. com frequência de $73,58 \%$. Estas relações são explicadas pelo fato de HA, DM e tabagismo acelerarem eventos ateroscleróticos, desenvolvendo placas mais instáveis e, consequentemente, oclusão arterial precoce e mais grave. A hipertensão, através da disfunção endotelial e do remodelamento vascular aumenta 

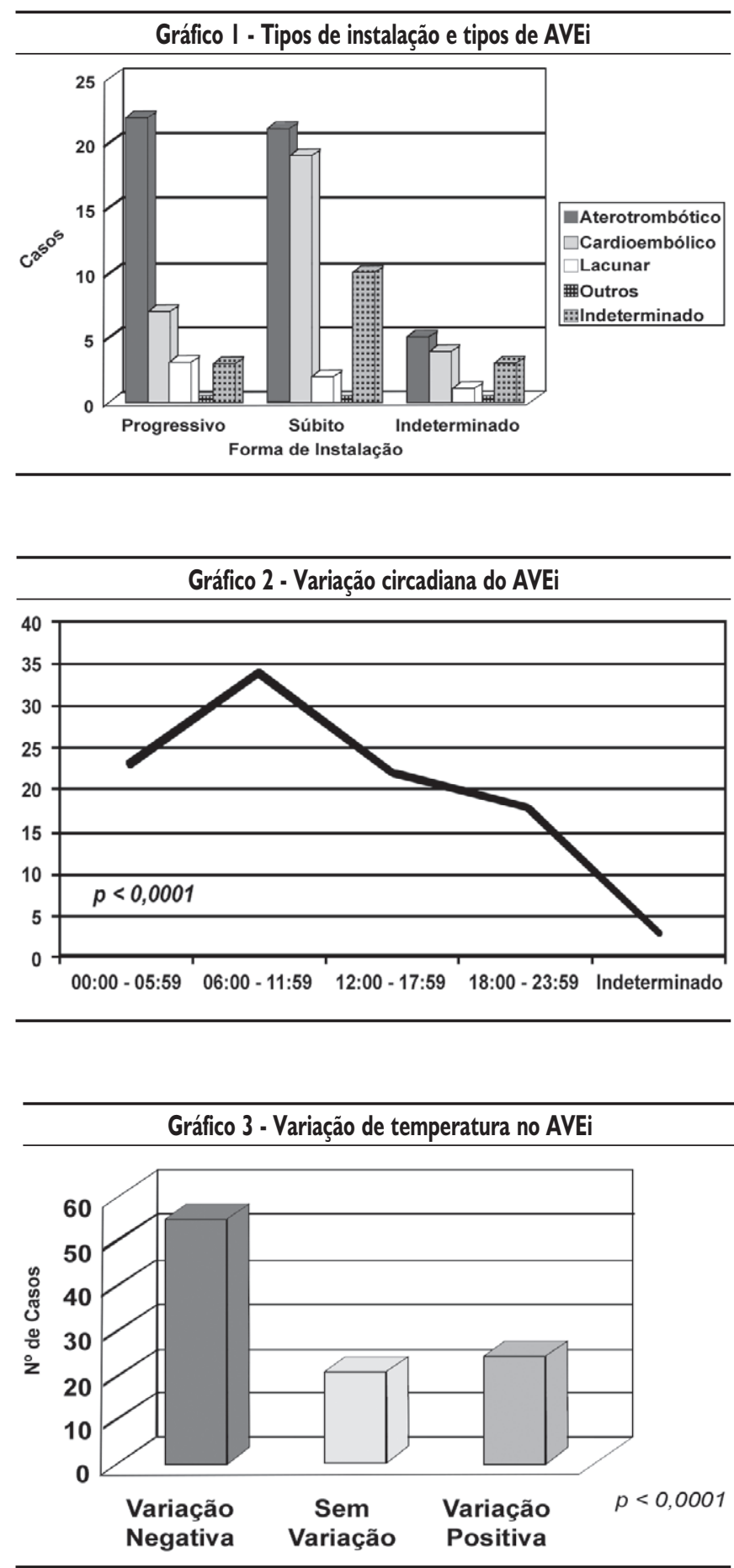

a formação de fatores de crescimento e citoquinas, permitindo a oxidação do LDL, recrutamento de leucócitos e redução da produção de substâncias protetoras, como o óxido nítrico, a prostaciclina. A disfunção endotelial também torna o leito arterial comprometido altamente propenso à vasoconstricção e ao vasoespasmo. Diversos estudos mostraram que uma glicemia superior a 130 - $140 \mathrm{mg} / \mathrm{dL}$ aumenta a mortalidade tardia no AVE isquêmico, mensurada nos primeiros 30 dias, I ano e 6 anos. Os efeitos da hiperglicemia sobre a área de isquemia e infarto cerebral podem ser variados: aumenta permeabilidade da barreira hematoencefálica e eleva o risco de degeneração hemorrágica. lá as cardiopatias favorecem a estase e turbulência sanguíneas intracardíacas e arritmias, podendo causar eventos cardioembólicos ${ }^{5-23}$

Ao avaliar o horário de instalação, encontrou-se 65,3\% de AVEi entre $06 h 00$ e I Ih59 ( $<<0,000$ I), sendo condizente com a literatura atual. Lago et al. encontraram, em seu estudo, que dentre os 924 pacientes internados por AVEi, 25,5\% referiram início dos sintomas ao acordar ou às 6h00. Oliveira et al. demonstraram que 39,62\% (21 pacientes) dos casos ocorreram entre $6 \mathrm{~h} 00$ e 12h00. Gupta et al. observaram que 47\% dos AVEi ocorreram entre 6hOl e I 2h00. Da mesma maneira, Gur et al. verificaram que dos 2.312 casos de AVEi, $34 \%$ ocorreram entre 6 h00 e IOh00.

$\mathrm{Na}$ análise da variaçãa de temperatura obteve-se que a redução na temperatura ambiente, em um período de 24 horas, foi considerado fator responsável pelo aumento na ocorrência dos eventos (55\%, $p<0$, 000 I), estando de acordo com Yun-Chun Hong et al. que estudaram 545 pacientes em três anos com dois grupos, um controle e outro exposto às variações de temperatura. Eles obtiveram como resultado um odds ratio de 2,9, com intervalo de confiança de $95 \%$ igual a I,5 ou 5,3, para uma variação negativa na temperatura, sendo o período de maior risco o de 24 horas. Concluíram, portanto, que mesmo baixas sutis de temperatura podem aumentar o risco de AVEi.

É referido que principalmente nas primeiras horas do dia, tônus simpático (consequentemente frequência cardíaca e pressão arterial), níveis de cortisol e insulina, agregação plaquetária e viscosidade sanguínea estão aumentados ${ }^{5-23}$. Da mesma maneira, fatores protetores, como atividade fibrinolitica, estão reduzidos neste período do dias-23. Além disso, foi demonstrado que nas épocas mais frias do ano, pressão arterial, número de plaquetas e hemácias, viscosidade do sangue e níveis de fibrinogênio e colesterol estão 23\% mais elevados, principalmente em indivíduos idosos ${ }^{5-23}$ É reconhecido também que a incidência de eventos isquêmicos aumenta nos períodos do ano em que são registradas as temperaturas mais baixas ${ }^{16}$. Em adição, no inverno pode ser observado aumento no número de internações hospitalares devido ao infarto agudo do miocárdio ${ }^{5-23}$.

\section{ConClusão}

A HA foi considerada, neste estudo, principal antecedente pessoal relacionado com AVEi. Notou-se, também, uma forte relação entre a prevalência de eventos encefálicos isquêmicos e variação negativa de temperatura. Por fim, o horário de maior frequência foi entre 6 h00 e I l h59.

Neste estudo não foi possível avaliar as variações de temperatura e circadianas para cada subtipo de AVEi, pois o número de casos estudados não permitiu avaliação estatística significante.

Portanto, acreditamos que um maior aprofundamento dos estudos sobre a variação de cada um destes fatores possibilitará o desenvolvimento de melhores programas de prevenção; de medidas que proporcionem um diagnóstico precoce; novas e mais eficazes estratégias de terapêutica com redução da morbimortalidade dos eventos vasculares encefálicos. 


\section{Agradecimentos}

Aos queridos professores, Dra. Dorotéia Rossi da Silva Souza e Dr. Moacir Fernandes de Godoy pela ajuda e dedicação.

\section{Conflito de interesse: não há}

\section{SUMMARY}

\section{INFLUENCE OF CIRCADIAN AND TEMPERATURE VARIATIONS ON THE ISCHEMIC STROKE}

BACKGROUND. Ischemic stroke (IS) ranks third as cause of death and annual costs. Literature shows a greater number of cases in the first hours of the morning and on colder days, allowing for better prevention at these times. The objectives were to identify risk factors, evaluate the influence of circadian and temperature variations on prevalence of IS.

METHODS. Onset time was analyzed during four periods and obtained from the patient or family. Weather temperature was obtained from the INMET. The IS were classified according to subtypes established by the TOAST project.

RESULTS. Hypertension was the main risk factor in $66 \%$ of the patients. There was a predominance of cases between 06:00 AM and I1:59 AM $(p<0.0001)$ and after a negative variation of temperature $(p<0.0001)$

CONCLUSIONS. A strong relationship was found between prevalence of $I S$ and negative variation of temperature. Events were more frequent between 6:00 AM and 11:59 AM. It was not possible to evaluate the variations for each subtype of IS because the number of cases did not allow for significant statistic evaluation. [Rev Assoc Med Bras 2009; 55(I): 60-3]

KEY wORDS: StROKE. Circadian rhythm. Temperature. Risk factors.

\section{REFERÊNCIAS}

I. Royal College of Physicians of London. National Clinical Guidelines for Stroke. Londres: Publications Unit of the Royal College of Physicians; 2004. p.3-10.

2. Rosamond W, Flegal K, Friday G, Furie K, Go A, Greenlung K, et al. Heart Disease and Stroke Statistics-2007 Update: A Report from the American Heart Association Statistics Committee and Stroke Statistics Subcommittee. Circulation. 2007; 1 15:69e-17/e.

3. Lavados PM, Hennis AJM, Fernandes JG, Medina MT, Legetic B, Hoppe $A$, et al. Stroke epidemiology, prevention, and management strategies at a regional level: Latin America and the Caribbean. Lancet Neurol. 2007;6:362-72

4. Classification of Subtype of Acute Ischemic Stroke. Definitions for use in a Multicenter Clinical Trial. TOAST. Trial of Org 10172 in acute stroke treatment. Stroke. 1993:24:35-41.

5. Nyquist PA, Wiebers DO, Crowson CS, O'fallon WM. Circadian and seasonal occurrence of subarachnoid and intracerebral hemorrhage. Neurology. 2001;56:190-3.

6. Oliveira HA, Moreira AJP, Oliveira AMP. Ritmo circadiano e doença vascular encefálica. Arq Neuropsiquiatria. 2004;62:292-6.

7. Gupta A, Shetty H. Circadian variation in stroke: a prospective hospitalbased study. Int I Clin Pract. 2005;59: I 272-5.

8. Hong YC, Rha JH, Lee JT, Ha EH, Kwon HJ, Kim HO. Ischemic stroke associated with decrease in temperature. Epidemiology. 2003; 1 4:473-8.

9. Kyobutungi C, Grau A, Stieglbauer G, Becher H. Absolute temperature, temperature changes and stroke risk: a case crossover study. Eur Epidemiol. 2005;20:693-8.
10. Chang CL, Shipley M, Marmot M, Poulter N. Lower ambient temperature was associated with an increased risk of hospitalization for stroke and acute myocardial infarction in young women. J Clin Epidemiol. 2004;57:749-57.

I I. Manfredini R, Boari B, Smolensky MH. Circadian variation in stroke onset: identical temporal pattern in ischemic and hemorrhagic events. Chronobiol Int. 2005;22:417-53.

12. Wang Y, Levi CR, Attia JR, D'este CA, Spratt N, Fischer J. Seasonal variation in stroke in the Hunter Region, Austrália: a 5-year hospital-based study, 1995-2000. Stroke. 2003;34: I |44-50.

13. Stergiou GS, Vemmos KN, Pliarchopoulou KM, Synetos AG, Roussias LG, Mountokalakis TD. Parallel morning and evening surge in stroke onset, blood pressure, and physical activity. Stroke. 2002;33: I 480-6.

14. Elliot WJ. Circadian variation in the timing of stroke onset: a meta-analysis. Stroke. 1998;29:992-6.

15. Chaturvedi S Jr, Woolson RF. Circadian variation in ischemic stroke subtypes. Stroke. 1999;30: 1793-5.

16. Kocer A, Ilhan A, Ince N, Bilge C. The related causes in very early morning onset of stroke. Prog Neuropsychopharmacol Biol Psychiatry. 2005;29:983-8.

17. Azevedo E, Ribeiro JÁ, Lopes F, Martins R, Barros H, Cold: a risk factor for stroke? J Neurol. 1995;242:217-21.

18. Gur AY, Bornstein NM. Are there any unique epidemiological and vascular risk factors for ischaemic strokes that occur in the morning hours? Eur J Neurol. 2000;7:179-81.

19. Smith EE, Abdullah AR, Amirfarzan H, Schwamm LH. Serum lipid profile on admission for ischemic stroke. Neurology. 2007;68:660-5.

20. Giorda CB, Avogaro A, Maggini M, Lombardo F, Mannucci E, Turco S, et al. Incidence and risk factors for stroke in type 2 diabetic patients. The DAl Study. Stroke. 2007;38:1।54-60.

21. Omama S. Yoshida Y. Ogawa A, Onoda T, Okayama A. Differences in circadian variation of cerebral infarction, intracerebral haemorrhage and subarachnoid haemorrhage by situation at onset. J Neurol Neurosurg Psychiatry. 2006;77: I345-9

22. Takekawa H, Miyamoto M, Miyamoto T, Hirata K. Circadian rhythm abnormalities in the acute phase of cerebral infarction correlate with poor prognosis in the chronic phase. Autonomic Neurosci: 2007; 13 1: 1 31-6.

23. Brown DW, Giles WH, Greenlund KJ. Blood pressure parameters and risk of fatal stroke, NHANES II Mortality Study. Am J Hypertens. 2007:20:338-41.

24. V Diretrizes Brasileiras de Hipertensão Arterial. Sociedades Brasileiras de Cardiologia, Hipertensão e Nefrologia; 2006. [citado out 2008]. Disponível em: http://www.sbn.org.br/Diretrizes/ V_Diretrizes_Brasileiras_de_Hipertensao_Arterial.pdf.

25. Milech, A; Forti, AC; Golbert, A, Gomes MB̈, Dib SA, editores. Tratamento e acompanhamento do Diabetes mellitus. São Paulo: Diretrizes da Sociedade Brasileira de Diabetes; 2006. [citado out 2008]. Disponível em: http://www. diabetes.org.br/educacao/docs/diretrizes.pdf.

26. II Diretriz Brasileira de Cardiopatia Grave. Arq Bras Cardiol. 2006;87:223-32.

27. Giannini SD, Santos RD, Fonseca FH, Moriguchi EH. III Diretrizes Brasileiras sobre Dislipidemias e Diretriz de Prevenção da Aterosclerose do Departamento de Aterosclerose da Sociedade Brasileira de Cardiologia; 2001. [citado out 2008]. Disponível em:http:// bvsms.saude.gov.br/bvs/publicações/diretrizcorreta.pdf.

28. Naradzay JFX. Transient ischemic attack. [cited 2007 Jan 10]. Available from: http://www.emedicine.com/emerg/topic604.htm.

29. Araújo, AJ; Menezes, AMB; Dórea, AJPS. Diretrizes para cessação do tabagismo. J Bras Pneumol. 2004:30( I):2-76.

30. Fuster $V$, Rydén MD, Asinger RW. ACC/AHA/ESC Guidelines for the Management of Patients with Atrial Fibrillation. J Am Coll Cardiol. 200 I;38: | 266ii- | 266lxx.

Artigo recebido: 21/01/08

Aceito para publicação: 24/05/08 